\title{
Comment articuler la linguistique et la sémiostylistique? \\ Le champ stylistique à l'épreuve de la matérialité de l'écrit
}

\author{
Marion Colas-Blaise \\ Université du Luxembourg, UR IPSE \\ UMR 7118, Université Nancy 2 \\ CeReS, Université de Limoges \\ marion.colas@uni.lu
}

\section{Introduction}

Pourquoi une linguistique de l'écrit? Quelles sont les demandes spécifiques adressées au linguiste, quels sont les modèles d'analyse qui permettent d'en capter la dynamique particulière ? Enfin, comment s'opère le glissement de l'écrit au texte littéraire, retenu ici, et à la reconnaissance d'un style littéraire ?

Un début de réponse est fourni par J. Gardes-Tamine (2004: 31): l'écrit constitue un «terrain d'observation beaucoup plus fécond que l'oral ». Il est crédité d'un atout de taille : soumettant à l'attention du linguiste des configurations sémanticosyntaxiques qui questionnent une conception normative de la langue et en éprouvent les limites, il offre un accroissement des possibilités d'analyse. J. Gardes-Tamine avance deux raisons au moins : un degré de maîtrise du projet d'expression plus important et la mise à contribution de la dimension spatiale.

Il semble ainsi possible de circonscrire le champ où opère une grammaire de l'écrit: une meilleure planification et gestion du déploiement du texte soumet à l'analyse une plus grande variété d'agencements à l'intérieur de la phrase, d'enchaînements interphrastiques responsables de la cohésion textuelle ou de configurations construisant la cohérence globale; enfin, la spatialisation peut non seulement soutenir la mémorisation discursive mais se charger de " valeurs symboliques » (ibid. : 38). En définissant le style comme «l'utilisation optimale et concertée des possibilités qu'offre la langue et non comme un écart par rapport à une norme, à supposer qu'on arrive à la fixer » (ibid. : 29), J. GardesTamine ménage le passage à l'écrit et au style littéraires, en le faisant apparaître, à la suite de C. Bally (voir aussi Adam, 1997 : 11), comme une différence surtout de degré.

En même temps, J.-M. Adam a montré que la différence entre la "grammaire» de l'écrit et la « linguistique » de l'écrit n'est pas que d'ordre lexical (ibid. : 21). Dans la perspective de l'Analyse de discours, le texte littéraire est appréhendé non seulement comme un " espace de réalisation de la langue », mais comme " un événement socio-discursif» (ibid. : 9-10), en relation avec le contexte de la production du discours, de sa circulation et de sa consommation. L'hypothèse à la base de cette réflexion est alors la suivante : si la dynamique de l'écrit peut être captée à travers l'agencement de la grammaire avec les approches de la linguistique textuelle, énonciative et pragmatique, les travaux de rhétorique et de poétique, il incombe au champ stylistique, à définir dans le cadre englobant fourni par l'Analyse de discours, d'accueillir la stratification des plans de structuration; allant à l'encontre d'une «démarche conjoncturelle de récupération et d'intégration-articulation œcuménique » (Adam, $2002: 72$ ), le récepteur gagne à réinterroger l'entrejeu des catégories d'analyse convoquées à partir d'un socle tensif.

Dans la première partie, l'accent sera mis sur les opérations qui, à partir du principe tensif, doivent permettre de rendre compte de la dynamique textuelle et discursive. Il s'agira, ensuite, d'instaurer un vaet-vient entre la réflexion théorique et l'étude d'exemples empruntés à Julien Gracq et à Michel Butor, en sélectionnant comme entrée pertinente une des spécificités de l'écrit: l'aménagement du support de l'écriture. On montrera en quoi la complexité de la construction de l'espace aux différents niveaux 
d'appréhension (le cadre phrastique, la page, le livre) et les formes de « marquage » du support (à travers les ressources typographiques) contribuent à la construction du sens et à la production d'effets de style.

\section{Le champ stylistique}

\subsection{Vers une définition du champ stylistique}

Selon J. Fontanille (1999 : 189), si le style apparaît comme une notion «préthéorique », qui appelle en tant qu'objet de connaissance une pluralité de points de vue subjectifs relevant plus ou moins de l'intuition, il est possible de réunir «un petit nombre d'alternatives et de traits communs » complémentaires, caractéristiques $\mathrm{du}$ «domaine à explorer». Ce sont les modalités de cette complémentarité que l'on vise à interroger ici, en proposant une définition du champ stylistique. Dégageant trois propriétés, on tentera de prendre, à chaque fois, la mesure de quelques-unes des conséquences épistémologiques et méthodologiques des choix opérés et des questions ainsi soulevées.

i) Le champ stylistique se construit à la faveur d'une interaction entre le sujet récepteur, doté d'une compétence linguistique et encyclopédique, et le texte, pourvu d'une morphologie déterminée, qui contraint plus ou moins sa réception ${ }^{1}$. Ainsi, le champ stylistique prend forme, selon des modes variables à déterminer, à partir du «champ de discours » constitué par le sujet d'énonciation en production, l'appréhension, à la fois sensible et cognitive, devant actualiser les potentialités en mettant au jour la congruence locale des traits structurants, voire la cohérence globale ${ }^{2}$.

Trois points méritent d'être examinés. D'abord, en mettant en avant les exigences de l'analyse immanente, la saisie du texte (de portions de texte) comme un ou des « touts de signification » a le mérite de poser la question de la fixation des limites. Celle-ci semble, en effet, être au cœur de la définition du champ stylistique relativement à un texte, c'est-à-dire en relation avec au moins une «concrétion en un certain sens matérielle, matérialisable en tout cas, ne serait-ce qu'en raison de l'exploitation de la substance de l'expression (le son, et les graphèmes sur la page) » (Molinié, 1994 : 205). En effet, comme nous y invitera la question de la matérialité du texte, le point de vue stylistique doit, à un moment donné, être articulé avec d'autres points de vue, notamment dans le cadre des "pratiques intersémiotiques » (Maingeneau, 1984 : 13). Ensuite, le champ stylistique doit être considéré comme traversé par des tensions renvoyant à des rapports graduables entre le local et le global, entre l'intra-, l'inter- et le contextuel. À partir d'un ensemble de marques identifiables dans le tissu textuel, l'analyse stylistique doit restituer et déployer les processus et les formations signifiantes à l'origine de déterminations ordonnables suivant un mouvement d'intégration croissante. Ainsi, l'analyse stylistique étant de type interactif, il faudra se doter des moyens conceptuels qui rendent possible une analyse rigoureuse des régimes de la réception, en fonction des «modes de présence » des « faits de style », qu'ils soient de nature textuelle, inter- ou contextuelle, au sein du champ stylistique.

ii) Conçu dans une extension large, le champ stylistique accueille des manifestations stylistiques qui peuvent être décrites à partir des oppositions croisées «singulier» $v S$ 《collectif» et « littéraire »vs « non littéraire » (ou « ordinaire »). La réflexion prend ainsi appui sur une conception « continuiste » de la langue au style (Jaubert, 2005 et 2007), qui met en avant le rapport dialectique entre les dimensions singularisante et universalisante du style ${ }^{3}$. De ce point de vue, il faut s'interroger sur les conditions auxquelles le style littéraire peut être appréhendé dans son devenir à partir d'autres ensembles signifiants, plus ou moins individualisants ou collectifs (cf. Adam, 1997).

On retiendra essentiellement deux questions. Celle de la littérarité, tout d'abord, que G. Molinié aborde en dégageant trois « composantes » considérées comme « définitoires de la littérarité » au niveau général :

Par un groupe de procédures qu'il faut poser et appliquer, on doit arriver à détecter des stylèmes de littérarité générale, qui différencieront le texte romantique du texte commercial. Ces procédures, en sémiostylistique, consistent à tester dans quelle mesure le discours analysé satisfait les trois composantes qui, dans cette théorie, sont définitoires de la littérarité : le discours littéraire (de l'intérieur) est son propre 
Dans le cadre d'une réflexion sur la réception des faits de langue comme " faits de style », on propose d'articuler la gradualité des «effets de littérarité » avec les «effets d'identité » dont le style est producteur ${ }^{4}$. Si l'on considère avec J. Fontanille (1999: 195) que le style combine des «identités textuelles », qui concernent le "texte, comme espace de distribution des effets", et des "identités discursives », en rapport avec le « domaine des valeurs, des modalités et des actes de langage ", la mise à nu des valeurs esthétiques, définitoires du texte littéraire ${ }^{5}$, mais aussi esthésiques et éthiques est du ressort de l'analyse stylistique plus que de celui d'autres approches, concurrentes.

La deuxième question ne concerne pas seulement la dimension collective ou particularisante du style, mais encore la nécessité d'articuler le «modèle stylistique» avec des modèles d'explication qui débordent le texte et le subsument: il faudra ouvrir le débat en direction, d'une part, de modèles généralisables dont les textes littéraires sont des actualisations parmi d'autres et, d'autre part, de styles ou « formes de vie ».

iii) L'interaction entre le sujet lecteur et l'objet-texte prend la forme de stratégies d'analyse mettant en œuvre des régimes, à définir en intensité (affective) et en étendue textuelle (saisie cognitive), spécifiques. Le champ stylistique est ainsi conçu comme un champ tensif. En effet, du point de vue de son organisation topologique, il comporte, comme tout autre champ, un centre ou noyau, mais aussi des zones plus ou moins périphériques, des horizons ou frontières en direction du hors-champ ; il est à géométrie variable, avec des frontières et des parois internes, dont la mobilité est fonction des régimes retenus.

Ces points seront examinés à la lumière du modèle tensif développé, surtout, par J. Fontanille (1998, 1999 ; Fontanille \& Zilberberg, 1998) et à partir des propositions d'A. Herschberg Pierrot : « Percevoir le style en tension, c'est considérer le texte de l'œuvre comme du continu, un continu actionnel et réactionnel avec soi-même et avec les autres textes » (2005: 44). Adoptant un point de vue méta-critique, on appliquera le principe tensif à la pratique stylistique elle-même, en s'interrogeant sur le choix des stratégies d'analyse au contact des faits de langue ou de style. L'hypothèse est qu'en mettant en avant les « réglages » interactionnels entre le récepteur et l'objet d'analyse, le cadre conceptuel tensif permet une approche unifiée de l'entrejeu des différentes dimensions du texte au sein du champ stylistique et des pratiques d'analyse suscitées et autorisées.

\subsection{Le principe tensif : les stratégies d'analyse et leurs régimes}

Ainsi que le souligne J.-M. Adam (1997 : 10), les textes littéraires peuvent apparaitre, du point de vue de la linguistique, comme des lieux privilégiés de manifestation des potentialités de la langue, où se déploient des "variations infinies». La question n'est pas seulement de réfléchir à l'apport de la linguistique à la stylistique littéraire ${ }^{6}$, mais de s'interroger sur le « supplément » de sens ou de valeur que celle-ci confère à celle-là : comme le note J.-M. Adam (ibid. : 9), «l'adoption d'un point de vue linguistique sur les textes littéraires doit entrainer une véritable reconception de la stylistique et l'attention au style, en retour, une redéfinition de la grammaire elle-même ».

Approchée sous cet angle, l'étude stylistique est pourvue, d'emblée, d'une visée : il s'agit de mettre en évidence des relations entre les faits de langue qui, de l'unité inférieure au mot ou au syntagme, à la construction morpho-syntaxique, aux enchainements interphrastiques, à tel type de progression textuelle, trouvent à signifier au sein du champ stylistique. Le prélèvement et le regroupement de faits pertinents peut paraitre malaisé : le style échappe, selon C. Metz (1991), à l'activité métadiscursive déployée par l'énonciation et se résume à une "manière d'être » plus ou moins diffuse, plus ou moins discrète ${ }^{7}$. Les principes guidant le prélèvement des éléments n'en sont pas moins énoncés avec force. Combinant forme et substance du contenu et de l'expression, G. Molinié décline quatre composantes du style :

a/ au niveau de la substance du contenu, une unité globale très partagée; b/ des structures fantasmatiques, imageantes récurrentes, ce qui nous conduit sans doute dans la forme du contenu, de même que c/ des schémas rhétoriques expressifs ; 
d/ enfin, au niveau de la forme de l'expression, un stock lexico-figuré et des tours de phrase, ce qui correspond, dans la tradition rhétorique, à l'élocution, c'est-à-dire exactement à la définition rhétorique du style (1994:205-206).

On peut encore citer N. Batt $(1997: 128)$ au sujet des quatre conditions de réussite d'une lecture « artistique » selon Lotman : pour mettre en œuvre une «corrélation dynamique », elle nécessite i) une indétermination de départ, qui peut correspondre aux «blancs » d'Iser, ii) la reconnaissance d'une base structurelle abstraite, iii) l'établissement d'un rapport entre oppositions aux niveaux concernés et iv) la réduplication des processus à un ou plusieurs niveaux d'analyse. Enfin, en ce qui concerne les choix méthodologiques, la lecture du texte littéraire requiert en même temps que la compréhension de l'ensemble, une dissociation et une autonomisation des éléments qui entrent en contact les uns avec les autres, auxquelles on ajoutera les opérations de la commutation et de la catalyse ; enfin, les éléments sont réassociés, à la faveur d'une opération de translation, dans des configurations originales.

Les exigences liées à l'analyse stylistique se précisent d'emblée : elle doit éviter le risque d'un rattachement presque mécanique des traits isolés aux différents niveaux à un schéma de base les subsumant. Le champ stylistique doit être conçu de telle sorte que la circulation du sens soit préservée, que des prédications imprévues, la dynamique d'un processus de réajustement ou de re-construction toujours à l'affût des indéterminations ou des inconsistances décelées rendent compte du style comme «processus », en préservant «la possibilité de hiérarchies mouvantes, de principes organisateurs en tension les uns avec les autres » (Herscherg Pierrot, 2005 : 43). D'où l'importance de la notion de variation $^{8}$ que $\mathrm{G}$. Molinié intègre dans sa définition du stylème :

On peut fixer un type d'objet décrit, et faire défiler tous les systèmes expressifs rattachables au même principe de le décrire, ou fixer un système expressif parmi le tout répertorié, et faire défiler la plus grande quantité de types d'objets décrits, chacun muni du trait spécifique de la catégorie (1994: 204).

D'où l'intérêt d'une approche tensive qui, en plaçant le principe de la gradualité ${ }^{9}$ au cœur de l'interaction entre le sujet récepteur et l'objet d'analyse, permet d'appréhender des «événements » et des «faits de style » dans un cadre conceptuel unifié, en en examinant le «mode de présence » au sein du champ stylistique. La définition du « fait de style » proposée par J.-M. Adam sert de base à la réflexion :

Par rapport à la reprise d'un ensemble de traits microlinguistiques qui caractérise, selon moi, un style, je définirai un fait de style comme un fait ponctuel de texture attendu ou inattendu au regard du style d'une œuvre, d'un auteur, d'un genre ou d'une école donnés. Un fait de style est donc le produit perçu d'une récurrence ou d'un contraste, d'une différence par rapport à des régularités micro-linguistiques observées et attendues d'un texte, d'un auteur, d'une école, d'un genre (1994:19).

On voit ici s'esquisser le continuum sur lequel se déploient les différences de saillance et de densité des faits de langue retenus, en fonction de leur fréquence et de la qualité ressentie en réception. Grâce au principe tensif, on peut montrer en quoi ces différences commandent, en partie, les stratégies d'analyse du récepteur ${ }^{10}$. D'un côté, on rend compte de l'action exercée par les « faits de style » sur le récepteur, dont ils captent l'attention selon des régimes à intensité variable et dont ils contraignent plus ou moins l'interprétation ${ }^{11}$; de l'autre, il est possible de décrire les degrés de l'implication vive du récepteur (axe de l'intensité), mais aussi les efforts déployés, en fonction de sa compétence linguistique et encyclopédique et en fonction du degré d'accessibilité des faits retenus, pour construire une cohérence (l'axe de l'étendue concerné par les degrés de l'élaboration cognitive). Globalement, la réception fait émerger des indices localisables à différentes strates, pour les nouer en faisceaux et construire une cohésion et une cohérence plus ou moins strictement poursuivies. La mise en évidence des régimes à partir des corrélations entre les dimensions continues de l'intensité sensible (perceptive ou affective) et de l'étendue (saisie cognitive) permet alors de concilier deux points de vue : celui des «effets d'identité »" produits par les faits de langue convertis en « faits de style » et celui des «styles » d'analyse propres aux récepteurs, l'essentiel se jouant à leur point de rencontre, là où se négocient les stratégies qui les mettent en œuvre. Par ce biais, l'approche tensive prend en considération le volet perceptivo-cognitif et sensible de l'analyse stylistique. 
Ouvrant l'éventail des positions stratégiques, on peut décliner au moins quatre régimes de la réception différents ${ }^{13}$. Contrant sans doute le danger d'une circularité qui voudrait que les faits de langue sélectionnés soient appelés à légitimer la base qu'ils ont façonnée, le régime englobant se saisit de « tous les détails » d'un texte ${ }^{14}$ et révèle un «effet d'identité » fort (intensité et étendue fortes). Pour que le degré de saturation maximale soit atteint, sans doute faut-il que soient également pris en considération le côté palpable (sonore, visuel...) des signes, la matérialité du support de l'écriture, voire, plus généralement, l'adéquation entre le dit et le dire, quand, comme l'écrit D. Maingeneau (1999: 80), «les "idées" se présentent à travers une manière de dire qui renvoie à une manière d'être, à la participation imaginaire à un vécu ». Face au «plein»d'indices, M. Riffaterre (1971:144) considère, pour sa part, qu'un «pas décisif a été fait vers la solution du problème du style lorsque [...] au lieu d'étudier également tous les aspects d'une structure, on s'est limité à ceux dont la perception est imposée au destinataire de l'acte de communication». On ajoutera qu'un fait de langue circonscrit peut être «saillant» et être perçu comme un «événement stylistique (intensité forte, étendue restreinte), mais aussi passer inaperçu (intensité et étendue faibles); de même, les occurrences multiples d'un fait de langue dans l'espace-temps du texte peuvent produire un effet cumulatif (intensité assez forte et prise en considération de vastes étendues textuelles) ; il se peut, enfin, que l'impact faiblisse proportionnellement à l'augmentation du nombre des répétitions (intensité faible et étendue forte).

Sur le fond d'une dialectique des projets de singularisation et des pressions exercées par les données socioculturelles et les codifications génériques, on se donne ainsi les moyens de rendre compte des positions s'échelonnant entre deux pôles : l'attendu, ce qui conforte l'« effet d'identité » produit par une œuvre, et la surprise ponctuelle. Dans le premier cas, la stratégie stylistique doit être englobante : elle doit rendre compte de la congruence des configurations structurantes avec un effet d'adéquation forte à l'œuvre, même si l'implication affective est réduite ${ }^{15}$. La surprise ponctuelle, quant à elle, bouleverse les modèles de prévisibilité. Elle risque d'être a-signifiante, dès lors que la stratégie d'analyse, particularisante, se satisfait d'un détail.

Du point de vue des phénomènes interdiscursifs et intertextuels, percevoir le champ stylistique comme un champ sous tension, c'est focaliser l'attention sur la zone de transition ou de transit où sont logés des apports discontinus, plus ou moins explicites et donc plus ou moins aisés à circonscrire et à identifier. Globalement, on conçoit l'intérêt de la notion de praxis que J. Fontanille définit ainsi :
[La praxis énonciative] n'est pas l'origine première du discours ; elle présuppose autre chose que l'activité discursive (le système de la langue, mais aussi l'ensemble des genres et des types de discours, ou des répertoires et encyclopédies de formes propres à une culture) ; elle suppose aussi une histoire de la praxis, des usages qui seraient des praxis antérieures, assumées par une collectivité et stockées en mémoire (1998:272).

Elle renoue avec le principe dialogique de Bakhtine et avec l'idée d'un fonds constitué par une hétérogénéité (une interdiscursivité et une intertextualité) jugée constitutive. Surtout, son soubassement tensif permet de rendre compte des degrés de l'assomption (intensité) des mots de l'Autre et de leur déploiement par reprise dans l'étendue du texte d'accueil. Les régimes de l'élaboration stylistique doivent ainsi s'ajuster aux régimes de la reprise. Dès lors qu'il s'agit de mettre l'accent sur l'emprunt et les modulations de sa valeur « selon ses contours plus ou moins nets, selon la souche dont il est issu et selon son point d'insertion » (Jaubert, 1990 : 147), rendre compte du « mode d'existence » en termes de degrés d'intensité et d'étendue permet de montrer, par exemple, comment le choix de la stratégie d'analyse est déterminé par la présence de l'allusion qui, en tant que « reprise non explicite de segments de linéarité [relevant] de la modalité autonymique » (Authier-Revuz, $2000: 210$ ), combine l'intensité assez forte liée à l'« auto-représentation opacifiante » avec une étendue plus vague ou diffuse que pour la citation. Afin de dégager les dynamiques enclenchées, il importe ainsi de confronter l'allusion, qui fait résonner d'autres occurrences déposées dans la mémoire intertextuelle ou interdiscursive du récepteur et sollicite la collaboration interprétative « en creux », avec la citation, qui porte à un degré suprême l'intensité vive de l'«en-soi » étranger, auquel le texte d'accueil réserve une portion d'étendue délimitée ; quant à l'« îlot textuel », il affiche son origine étrangère tout en bénéficiant d'une intégration forte dans le texte second ${ }^{16}$. L'approche tensive doit ainsi permettre d'esquisser des équilibres fragiles entre les variations intensives et 
quantitatives relatives à l'appropriation et à la remise en perspective de l'emprunt par l'énonciation seconde, et celles qui continuent à caractériser l'emprunt dans sa matérialité.

Enfin, ce même cadre tensif permet de caractériser les «modes de présence» des «faits de style » « contextuels », qui portent la marque des échanges plus ou moins continus et diffus avec le " horstexte $»$, avec les cadres socio-historiques, le contexte de la production, de la circulation ou de la réception, bref, avec les lieux où se décide le devenir des formes d'expression collectives, plus ou moins stabilisées et codifiées, où s'exerce l'arbitrage des «morales du langage » selon Barthes :

L'Histoire est $[\ldots]$ devant l'écrivain comme l'avènement d'une option nécessaire entre plusieurs morales du langage ; elle l'oblige à signifier la Littérature selon des possibles dont il n'est pas le maître. (1953 et $1972: 8$ ).

L'intérêt est non seulement d'accéder aux valeurs (éthiques, esthétiques) attachées aux régimes d'identité sélectionnés, mais encore de rendre compte, à côté des «morales du langage », de "morales de la réception » : c'est à partir des mêmes principes que l'on peut faire ressortir l'obédience au contexte idéologico-culturel des formes que revêt la stylistique (voir Maingeneau 2003 : 15-25) ainsi que celle des esthétiques; c'est par rapport à ces formes et esthétiques que se détermine la valeur d'un texte ou discours, voire celle de l'émotion esthétique, qui comprend elle-même une dimension collective. Si, en dernière instance, la conception du texte littéraire et la définition du champ stylistique, qui s'inspirent et s'étayent mutuellement, doivent être approchées comme des variables tributaires d'un contexte historique, le principe tensif permet de se rapporter à une histoire des formes et des postures critiques, au-delà de la « valeur instrumentale », au-delà même de la valeur intrinsèque du modèle d'analyse, et de la « justesse » relative à un point de vue.

\subsection{La modélisation méta-stylistique : invariant et variation}

Dans quelle mesure le point de vue stylistique gagne-t-il à être articulé avec d'autres points de vue, « externes »? Dans quelle mesure l'analyse qui privilégie le champ stylistique autorise-t-elle, voire appelle-t-elle à dégager, à travers les marques textuelles observables, une activité réflexive qui exige la prise en considération d'autres niveaux de pertinence? Si l'on admet qu'à ces niveaux s'élaborent des modélisations plus générales, au regard desquelles le texte et le style relatif à une forme singulière idiosyncrasique apparaissent comme des variantes, il paraît utile de confronter les préoccupations du stylisticien avec les travaux sur les régimes réflexifs du discours distingués par J. Fontanille (2003). Pour cerner davantage la spécificité de l'analyse stylistique, médiatisée par le champ stylistique, on note ainsi que l'élaboration, par "conversion $»^{17}$, d'une signification d'ensemble cohérente, investie de valeurs esthésiques, éthiques et esthétiques et productrice d'"effets d'identité », est rattachée au poste de la « sémiotique connotative», où la variation est mise au service de la stabilisation intentionnelle d'un invariant identifié ${ }^{18}$. Si l'on admet ainsi l'existence d'une "stylistique connotative », qui rend compte indirectement de la gradualité de l'«appropriation » de la langue selon A. Jaubert (un style, $d u$ style, le style ; 2005, 2007), on dira qu'elle se distingue à la fois de l'ébauche de modèle, qui est de l'ordre de la paraphrase - J. Fontanille parle dans ce cas de « modèles ad hoc, de simulacres qui servent de support ou d'alibi à l'intuition » $(2003: 121)$ - et des modèles généralisables, de type méta-stylistique ${ }^{19}$, qui transforment le texte (l'invariant fourni par les procédés morphosyntaxiques, les figures et thématiques...) en une variante possible. Dans ce cas, le texte n'endosse pas qu'une fonction d'illustration ou de validation du modèle ; il agit sur lui en retour, l'infléchit et le déforme.

Que le stylisticien ne puisse se désintéresser du devenir du modèle stylistique, de sa possible « conversion » en un modèle plus général, l'analyse de «crises alternatives », selon l'expression de J. Fontanille, ou encore de la survenue d'un «événement de style » en un point du texte l'atteste avec éclat. On songe ainsi aux détournements de proverbes analysés par A. Grésillon et D. Maingeneau (1984: 121): ils montrent comment la suspension des valeurs établies, une remise en question des comportements consacrés par l'habitude, mais aussi une remontée dans le sentir grâce à des propositions innovantes rejaillissent avec éclat sur le genre discursif, qui n'est pas dissociable de la pratique du proverbe à l'intérieur d'un espace littéraire et social. Le cas peut paraître exemplaire, dans la mesure où, 
au-delà de la dimension critique, la « restitution des possibles » peut inspirer un modèle méta-stylistique plus général : grâce à la «praxis énonciative » qu'il présuppose et qu'il alimente à son tour, ce modèle redéploie les modalités du fonctionnement de l'écriture proverbiale et les enjeux y relatifs; ïl la situe par rapport à d'autres formes d'expression non figées et subsume ses réalisations particulières.

Enfin, l'analyse stylistique doit-elle articuler le niveau de pertinence du texte, élu prioritairement, avec d'autres niveaux plus englobants (l'objet-livre, les pratiques et les «formes de vie », nécessairement « situés ») ? L'étude de cas, consacrée à des faits typographiques, rendra cette question plus pressante.

\section{Le champ stylistique à l'épreuve de la matérialité de l'écrit}

Pour mettre ces propositions à l'épreuve du cas concret, on se demandera, à partir d'extraits de Julien Gracq et de Michel Butor, sous quelles conditions spécifiques des procédés ayant trait à la spatialisation de l'écrit et à la matérialité du support peuvent être réexaminés à la lumière du champ stylistique et, plus largement, d'une pragmatique littéraire. En d'autres termes, sous quelles conditions, et à quels frais, les faits sélectionnés appellent-ils une interprétation qui, à partir d'une position critique elle-même historique, cherche à articuler cohérence idiosyncrasique et morale du langage, singularité du style et inscription socioculturelle, voire invite à un redéploiement «méta-critique »? Cela revient à détecter un double seuil : celui à partir duquel les procédés sélectionnés acquièrent un «effet stylistique » selon Riffaterre (op. cit. : 64), ou encore sont identifiés comme des «événements» ou comme des «faits de style », nécessairement précaires, qui résultent de la dynamique du texte ; enfin, le seuil à partir duquel, en fonction du régime mis en œuvre, les « événements » et « faits de style » prennent toute leur pertinence à hauteur de l'espace littéraire ou culturel et par rapport à des modèles qui débordent la dimension connotative du discours et la pratique analytique correspondante.

L'attention accordée à la forme matérielle de l'écrit (Charaudeau \& Maingeneau, 2002: 558) - en l'occurrence, le tiret simple ou double, l'italique et l'organisation du champ graphique - présente un triple intérêt. D'abord, elle permet de pointer certaines des marques textuelles à la base des différents régimes de la réception stylistique; porteurs de valeurs générales (cf. notamment Drillon, 1994), les signes typographiques constituent des candidats potentiels à une entreprise de «déformation » et de modélisation à portée plus large, qui rouvre l'éventail des variétés et enclenche une réflexion sur les systèmes de représentation. Ensuite, montrer en quoi les supports de l'écrit participent à la construction du sens, c'est envisager la nécessité de la linguistique de l'écrit sur laquelle nous sommes invités à réfléchir. Enfin, les signes typographiques, mais aussi les «modulations spatiales» (les marges, les paragraphes, les choix de police...) incitent à une appréhension elle-même plurielle : d'un côté, on dira que les aspects matériels et sensibles sont renvoyés à la substance de l'expression et que seule l'exploitation dans un texte les investit de formes de contenu et de valeurs; de l'autre, la «face» matérielle du texte selon J. Fontanille (2006: 221) exige que soit également pris en compte le « niveau suivant»- en l'occurrence, celui de l'objet-livre -, auquel le texte «fournit les premiers éléments sensibles et matériels $\gg$.

Soit d'abord l'emploi du tiret, qui fait l'objet, chez J. Gracq, d'un commentaire méta-critique : «C'est pour certains le génie de notre langue de n'ajuster sa phrase que par boutons et boutonnières, et de traquer à mort l'amphibologie, avant tout à titre de laisser-aller ", écrit-il dans En lisant en écrivant, avant d'ajouter : «Et si ma pente naturelle est de donner à chaque proposition, à chaque membre de la phrase, le maximum d'autonomie, comme me le signale l'usage croissant des tirets, qui suspendent la constriction syntaxique, obligent la phrase à cesser un instant de tendre les rênes ? » (1995 : 734-735).

Se trouve ainsi soulignée la nécessité d'une exploitation plurielle de l'emploi du tiret simple et double, ce que confirme le classement de celui-ci parmi les « ajouts » (Authier-Revuz \& Lala, 2002 : 7-12) : en prise sur plusieurs types de fonctionnement - typographique, syntacticoénonciatif, pragmaticosémantique, textuel - le décrochement typographique affiche son caractère transversal et réclame plus que d'autres phénomènes l'articulation des dimensions micro- et macroscopiques jugées complémentaires. Dans la perspective d'une pragmatique littéraire intégrant la notion de champ stylistique, la partition ostensible de l'espace phrastique ou textuel à travers la rupture, unique ou répétée, de la linéarité du signifiant, 
demande ainsi à être approchée sous différents angles. Appuyé sur un sous-ensemble de textes fictionnels unitaire (les romans Le Rivage des Syrtes et Un balcon en forêt), un parcours heuristique mène de considérations morphosyntaxiques et sémantiques dans l'ordre de la phrase à une approche textuelle focalisant l'attention sur les liens transphrastiques, la dynamique informationnelle et une analyse du mouvement de l'énonciation en acte. Dans les limites de cette étude, on déclinera les points de vue succinctement, mettant l'accent sur la congruence des sélections syntaxiques et sémantiques à différents niveaux et l'entrejeu des dimensions textuelles orchestré au sein du champ stylistique.

Ainsi, privilégiant d'abord les emplois du tiret double, on se contentera de dire qu'il participe, par sa valeur générale, d'une logique de la rupture et de la reprise, grâce au fonctionnement sémanticosyntaxique complexe de l'élément inséré, dont le décrochement typographique rend visible l'«accessoirité » syntaxique (Boucheron-Pétillon, 2002: 124). Ainsi, qu'il soit de nature endo- ou exophrastique, c'est-à-dire qu'il renvoie à une portion du monde référentiel ou qu'il soumette l'énoncé d'accueil à un commentaire, un jugement (Guimier, $1996: 6$ ), l'élément enserré par les tirets - de nature adverbiale ou adjectivale au sens large, nominale (avec/sans déterminant ou expansion) ou propositionnelle - a, du point de vue de la valeur sémantique, un statut surplombant, non seulement «méta-phrastique » (avec une opacification du sémantisme comme dans la modalisation), mais encore « inter-phrastique »; enfin, du point de vue de son mode de fonctionnement syntaxique, le décroché est de type à la fois extra-prédicatif (comme tout adverbe exophrastique) et «inter-prédicatif». Ainsi, malgré l'instanciation lexicale qui, à moins d'une véritable rupture thématique, rend possibles les appariements sémantiques au-delà même des bornes matérialisées par les tirets, il fait entrer en résonance deux espaces sémanticosyntaxiques autonomes, la base phrastique et un espace «hors phrase $»^{20}$. En même temps, l'autonomie n'a d'intérêt que corrélée avec l'élan, qui combine inchoativité et mouvement, la rupture et la continuation conquise sur l'arrêt. Elle doit être mise à profit par une énonciation en acte, une visée pragmaticosémantique et un dynamisme communicatif qui élargissent le champ de l'interprétation. Ainsi, à la faveur d'un fonctionnement ana- et/ou cataphorique, l'énergie nouvelle peut se propager au-delà même des bornes du cadre phrastique, en faveur d'enchaînements incertains, voire d'une véritable plurivocité interprétative ${ }^{21}$. Le décrochement typographique, qui est de nature autodialogique, peut accueillir, de surcroît, une pluralité de voix.

Dans les limites de cette réflexion, l'essentiel concerne, d'une part, l'intérêt pour la grammaire d'une étude du tiret double ou simple chez Julien Gracq, et, d'autre part, le passage de la mise à contribution des différentes dimensions du texte à l'exploitation stylistique; celle-ci la présuppose, tout en faisant valoir sa spécificité. En d'autres termes, à quelles conditions le tiret double doit-il satisfaire pour être hissé au rang de « fait de style » contribuant à produire des « effets d'identité »? On en retiendra deux. Il faut d'abord que les valeurs associées aux différentes occurrences puissent être érigées en invariant : on peut dégager, en l'occurrence, un schème structural très général, dont les oppositions rupture/reprise, blocage/réouverture constituent les versants temporel et spatial, et dont les opérateurs de l'implication (si... alors) et de la concession (malgré; la phrase se poursuit malgré la rupture) (Zilberberg, 2006) fournissent une version processuelle. S'arc-boutant sur une même base structurale, le tiret simple obéit, pour sa part, à une logique non plus de l'insertion, mais de l'intégration, qui met en œuvre une progression de type cumulatif, par à-coups et tâtonnements successifs. Il s'agit, ensuite, de tester la propension du tiret à "faire système» avec une liste close d'éléments expressifs, de thématiques ponctuelles, de procédés syntaxiques ou d'autres ponctèmes, susceptibles de se rattacher à cette même base.

Il importe alors de rendre compte des «modes de présence » variables que le tiret, simple ou double, se voit accorder au sein des champs stylistiques, et donc de la part qu'il prend dans la production d'« effets d'identité ». Soient ainsi, concrètement, les hésitations, dans tel passage du Rivage des Syrtes, entre une écriture par décrochement et une écriture dé-liée :

(1) $[\ldots]$ un instant du monde dans la pleine lumière de la conscience a abouti à eux [les hommes sur lesquels s'est portée l'attention de tout un peuple] - un instant en eux l'angoisse éteinte du possible a fait la nuit - le monde orageux de millions de charges 
Elles suscitent un questionnement lui-même double : sommes-nous face à un développement extensif, amorcé par le tiret inaugural et clôturé par le tiret final, qui se greffe sur la base insérante et la commente en dépliant le contenu sémantique du verbe mis en italique ? Il prend lui-même la forme de deux segments homofonctionnels ou donne lieu à un décrochement interne. Dira-t-on, au contraire, que la progression, heurtée, ajoute des tirets simples qui, malgré les déchirures du tissu textuel, tendent vers une sommation intégrative que la fin ne procure pas? L'articulation du questionnement linguistique avec des préoccupations stylistiques exige un déplacement d'accent. On se demandera plutôt si l'hésitation entre deux logiques de l'«amplification» (Gardes-Tamine, 2004) est pertinente au regard de la totalité signifiante : dans ce cas, à travers une tension que l'on peut ramener à la différence entre une attitude réflexive, qui privilégie l'explication métadiscursive, et une position de retrait du sujet, qui se soumet au flux de ses pensées, les emplois du tiret, simple ou double, proposent une forme d'analyse et de commentaire du «tiraillement » entre deux façons d'être-au-monde, qui est également pris en charge à d'autres niveaux de pertinence.

De fait, suivant les textes, une même base structurale accueille des investissements figuratifs et thématiques différents. Dans Le Rivage des Syrtes, les emplois du tiret double et simple s'interprètent sur le fond constitué par le sursaut d'un peuple qui, fédérant les initiatives éparses, secouant la léthargie ambiante, cherche à se donner un «destin » ou une «destination»; de concert avec la convocation et le détournement de motifs ou de scénarios plus ou moins stéréotypés, fixés par la tradition (la messe de Minuit et la naissance du Christ, la valorisation de l'inchoativité présente dans l'«aube » et dans le franchissement critique de la frontière...), le décrochement typographique contribue à signifier la rupture et l'irruption de la nouveauté. En même temps, il incombe au tiret fermant, mais aussi, plus largement, à la compétition entre le tiret double et le tiret simple, liée à l'entrechoquement de deux logiques concurrentes, de signaler immédiatement l'emprise du modèle historicisant et d'une nécessité qui échappe au contrôle du sujet et le conduit à sa destruction.

Dans Un balcon en forêt, la discrétion relative du tiret double contraste avec l'insistance du tiret simple, dont les emplois méritent d'être mis en relation avec la «poétique » de l'intervalle qui vise, non plus à remettre l'histoire en marche, mais à permettre au protagoniste de s'absenter de la guerre pour un temps et d'habiter l'intervalle à sa guise :

(2) Naturellement, ce n'est pas la ligne Maginot, songeait Grange, levant les yeux malgré lui vers les nids d'aigle broussailleux qui s'enlevaient très haut au-dessus de la rivière - mais en somme cette fortification paresseuse rassurait plutôt : visiblement on ne s'attendait ici à rien de sérieux. (Gracq, $1995: 25$ )

L'intéressant, du point de vue de la réception stylistique, c'est que le concours des niveaux de pertinence appelle, ici et là, des régimes différents. Dans Un balcon en forêt, le tiret forme système avec d'autres ponctèmes (le deux-points, le point-virgule...) pour proposer une reformulation, assumée par le locuteur, cohérente et prévisible, des tentatives d'organisation de l'espace-temps par le personnage. Récurrent sur de vastes étendues textuelles, il draine vers lui, pour s'y connecter, les isotopies figuratives et thématiques, les constructions syntaxiques auxquelles le schème structural peut être associé, et les noue ensemble au sein d'un système semi-symbolique qui appelle une stratégie d'analyse à fort pouvoir intégratif $\mathrm{f}^{22}$.

Dans Le Rivage des Syrtes, le tiret vaut davantage comme une forme de l'expression répondant à la définition rhétorique du style selon G. Molinié (1994: 206). Au-delà des analyses linguistiques fines, les associations sont de l'ordre de la "projection symbolique », plutôt que du "système semi-symbolique » (Fontanille, 2003 : 124-125). Redondante avec le contenu du texte, paraphrastique, la description - par exemple, «le tiret signifie l'irruption du nouveau dans la léthargie ambiante...»-est cantonnée dans la saisie de certaines des sélections spécifiquement liées à ce texte. Certes, le procédé est récurrent sur de larges pans textuels et il bénéficie d'une prise en charge méta-critique; cependant, ni saillant, ni 
intégrable directement dans la signification d'ensemble, il se prête à une stratégie d'analyse au mieux cumulative.

L'emploi de l'italique appelle des éclairages supplémentaires. Le mot italicisé met l'analyse stylistique au défi de rendre compte de forces "centrifuges » qui, plus que le tiret, donnent à voir l'hétérogénéité constitutive : dessinant des échappées du sens, elles favorisent la superposition, l'intrication ou l'entrée en conflit de plusieurs lignes thématiques. La stratégie stylistique appelée à s'emparer de ce « fait de style » doit ainsi combiner l'intensité vive d'un fait marquant avec une contribution relativement faible à l'établissement de la cohérence du « tout de sens ». En tant qu'événement énonciatif, en relation avec « le coup de théâtre de la trouvaille [de mot] » (Gracq, André Breton, 1989 : 506), le mot italicisé bouleverse, en effet, les ordonnancements linéaires sur le support de la page et fait résonner les mots marqués typographiquement «à distance ». Il en va ainsi du verbe "aboutir », dans l'exemple (1) (Le Rivage des Syrtes, 1989: 730) : il concentre sur lui les expressions en italique - "vitesse mentale », "fondre », « délivrer du mal », «arme du crime », «âmes damnées » ou «être » (ibid. : 729-730) - qui s'égrènent au fil des lignes précédentes et qui, ainsi rapprochées, se trouvent proposées à des voisinages inédits. À cela s'ajoute qu'à la faveur d'un flou conceptuel, des lexèmes tels que «fondre » «aboutir» ou « ailleurs » (ibid. : 735 ) non seulement libèrent l'« énergie latente en puissance dans le vocable » (André Breton, 1989 : 503), mais ouvrent sur l'épaisseur des relations intertextuelles. Enfin, le mot ainsi exhibé est non seulement dépouillé des collocations routinières, mais il est arraché au cotexte : "Nom, adjectif ou verbe, le mot considéré dans son isolement, "en liberté", polarise autour de lui comme de lui-même le meilleur de l'espoir de tout ce qui tend en nous à communier avec le monde [...]» (ibid. : 480). On voit comment, signifiant la crise et la « rébellion instantanée » du mot (ibid. : 504), l'italique inscrit dans le texte la nécessité d'un renouvellement de l'usage et dit l'urgence de la prise en considération des champs et espaces discursifs selon Maingeneau (1984). Les choix typographiques renvoient non seulement à un projet d'expression particulier, mais à une esthétique, à un style de vie, individuel ou collectif, plus ou moins identifiable, voire à une «forme de vie» (Fontanille, 2004 : 192). Celle-ci correspond au choix de valeurs dissidentes, qui remettent en question les codes discursifs établis et inventent un nouvel être-aumonde par le langage. En même temps, débordant les frontières du texte et du champ stylistique, la « forme de vie » peut introduire à une réflexion de nature méta-sémiotique, plus générale et plus abstraite, sur les possibilités offertes par différents systèmes de représentation, verbal ou non verbal.

Cependant, la prise en considération de la matérialité du signe demande aussi que soit explicitée la manière dont le «texte formel » accueille et fait signifier les signes en provenance d'un niveau inférieur, les «unités minimales» selon Fontanille (2006), qui sollicitent le canal sensoriel de la vue (lettres capitales, caractères minuscules, italiques ou romains, figures géométriques, espaces...). En attirant l'attention sur la manière de dire, le dédoublement méta-énonciatif constitutif de la modalité autonymique selon J. Authier-Revuz incite, en effet, à une saisie des propriétés spatiales et iconiques du signe italicisé, en tant que celui-ci renvoie au geste et au corps (la main qui incline les lettres) de l'instance écrivante. On en conçoit les enjeux : sous quelles conditions la prise en compte de la matière de l'expression relève-telle de l'analyse stylistique ?

Si dans Mobile (1962), Michel Butor porte l'expérience de l'écriture à un degré extrême, il est frappant de constater que la critique retient d'abord la fonction «mimétique» des ressources typographiques, subordonnées, globalement, à une logique du morcellement et de la recomposition de l'espace des ÉtatsUnis, et donc à une fonction de «représentation du sens » (Helbo, $1975: 87)$. On distinguera quatre cas. Ainsi, le blanc typographique est immédiatement investi de sens, quand il lui incombe, symboliquement, de «réfléchir » (Helbo, ibid. : 69) le problème géographique et politique frappant l'île d'Hawaï. Michel Butor lui-même commente ainsi sa pratique des compositions plastiques complexes du blason et de la cellule, qui abritent selon des lois précises des lettres capitales et des caractères minuscules, tantôt italiques, tantôt romains : "Dans les états [depuis le Texas et le Kansas jusqu'à la Caroline du Nord et la Floride] où il [l'élément "Noir"] apparaît j'ai toujours mis à la première apparition du blason, de cette constellation d'éléments, l'élément "Noir" à la fin en lui faisant remonter au cours du chapitre chaque fois une place parmi le nombre des éléments. Ceci produit, quand on lit le livre, une impression [...] de soulèvement » (Aubyn, 1964: 433). La «symbolisation» (Helbo, ibid. : 86), c'est-à-dire la mise à 
contribution des jeux de marges, des variations de police et de lignes, de la distribution des unités à l'intérieur des chapitres, qui corroborent l'« unité sémantique », implique une stratégie mettant en œuvre des valeurs d'intensité et d'étendue fortes. Les résistances à l'intégration sont d'autant plus remarquables : les rapprochements appelés par les homonymes, mais aussi les mouvements obliques du regard peuvent obliger à une prolifération des parcours de sens simultanés, proposés comme «en supplément »; ils sont cantonnés dans la page, qui devient le lieu d'inscription de relations signifiantes déconnectées du sens global, ou la débordent. Allégée du poids du symbolique, l'image iconique repose sur une relation de similarité entre le mouvement des marges et celui des vagues ou les effets de perspective produits. Enfin, le texte verbal porte les marques de l'influence picturale, en intégrant, par exemple, la figure géométrique du rectangle qui, écrit A. Helbo (ibid. : 88), revêt un « aspect "gratuit", "esthétique", sans portée réaliste ».

Ce dernier point mérite considération. En opposant une résistance à l'intégration dans une totalité interprétative, ces éléments invitent, en effet, à une confrontation qui, les marges des choix paradigmatiques et syntagmatiques étant débordées, interroge la cohérence de l'ensemble. Éprouvant les limites du faire sens, ces éléments posent la question générale de la mise à contribution des ressources matérielles de l'écrit et appellent, à ce titre, la conversion « méta-stylistique ».

Plus globalement, pour rendre compte des degrés de l'exploitation des ressources typographiques dans le cadre de l'analyse stylistique, on propose un double élargissement conceptuel : d'abord en direction de la notion d' "imagerie » fondée sur "une homologie entre les images mentales déclenchées par les représentations iconiques et celles suscitées par le verbal [...]» (Bonhomme, 2003 : 179); ensuite, en direction du concept d'intermédialité qui étudie, selon Méchoulan (2005), « comment textes et discours ne sont pas seulement des ordres de langage, mais aussi des supports, des modes de transmission, des apprentissages de codes, des leçons de choses » (cité par Badir, 2007 : 26-27).

Ainsi, dans une perspective pragmatico-rhétorique attentive à la réception interactive, l'intérêt du concept d'imagerie peut être lié à la gradualité des interprétations : i) les schèmes sensibles se satisfont d'une appréhension perceptuelle et émotive, esthésique avant d'être esthétique, d'une approche phénoménologique de l'énonciation; l'«évocation» est d'autant plus directe que l'image est conventionnelle (p. ex., la pointe de la flèche désignant le vol des oiseaux) et qu'elle est directement accessible du point de vue cognitif(intensité et extensité réduites); ii) si cette «tentation phénoménologique » correspond au degré zéro de l'analyse stylistique, la déhiscence des niveaux inscrit en creux la possibilité de l'interprétation ; c'est sur la base de l'interaction entre le récepteur et le texte et ses fonctions (épistémologique, analogique, esthétique...) que peuvent se négocier les régimes d'intensité et d'étendue mis en œuvre par l'élaboration d'un système semi-symbolique et que se détermine le degré de prise en charge de toutes ces productions signifiantes qui en défient les limites; il peut s'agir de ruptures figuratives ou thématiques ou l'analyste peut se trouver interpellé par la complexité d'un ensemble polymédial ou par les variétés de la translation inter- ou transmédiatique ${ }^{23}$.

Quant au concept d'intermédialité selon Méchoulan, on notera avec S. Badir (2007: 35) que l'analyse des discours rencontre l'analyse des médias : «[...] les œuvres sont toujours dépendantes d'un discours, mais elles dépendent toujours aussi, dans le même temps (dans le temps de l'analyse comme celui de l'appréhension phénoménale), d'un média ». Surtout, le concept d'intermédialité permet de penser l'articulation entre les deux « faces » du texte (Fontanille, 2006), le texte « formel», qui fournit un plan de pertinence aux signes, et le texte «matériel », qui ouvre sur le niveau englobant des objets : l'objetlivre et la pratique qu'il appelle, c'est-à-dire les usages qui en sont faits dans des situations socioculturelles et historiques données.

$\mathrm{Au}$ terme de ces investigations, on dira que dans la perspective d'une linguistique de l'écrit qui met en avant la notion de champ stylistique, l'intérêt de la question de la matérialité de l'écrit est au moins double. D'une part, le traitement appelé par l'exploitation des ressources typographiques paraît confirmer le rôle central joué par le champ stylistique, qui médiatise la réception, et la pertinence du principe tensif, grâce auquel il est possible, à travers une interaction entre les faits observables et le récepteur, de déterminer les degrés de littérarité ainsi que les «effets d'identité » produits. En même temps, les 
ressources typographiques signalent l'opportunité d'un dialogue entre le point de vue stylistique et d'autres points de vue : d'un côté, le point de vue méta-stylistique, de l'autre, des points de vue relatifs à d'autres niveaux de pertinence que le texte. Le «texte matériel » ouvre, en effet, sur un autre niveau d'expression, celui de l'objet-livre comme surface ou volume. En tant qu'espace d'une expérience littéraire ancrée dans un contexte d'actualité, le texte y est rendu signifiant à la lumière d'une méditation sur le live comme «objet complet » : «De l'objet de consommation au sens le plus trivial du terme, écrit Michel Butor, on passe à l'objet d'étude et de contemplation, qui nourrit sans se consumer, qui transforme la façon dont nous connaissons et nous habitons l'univers » (1964 : 137).

\section{Références bibliographiques}

Achard-Bayle, G. (2001). Grammaire des métamorphoses. Bruxelles : De Boeck \& Larcier.

Adam, J.-M. (1994). Style et fait de style : un exemple rimbaldien. In Molinié, G. \& Cahné, P. (éd.), Qu'est-ce que le style?, Paris : PUF, 15-43.

Adam, J.-M. (1997). Le style dans la langue. Une reconception de la stylistique. Lausanne : Delachaux \& Niestlé.

Adam, J.-M. (2002). Le style dans la langue et dans les textes. Langue française, 135, 71-94.

Aubyn, F. C. St. (1964). À propos de «Mobile » : Deuxième entretien avec Michel Butor. French Review, 38, 427440.

Authier-Revuz, J. (2000). Aux risques de l'allusion. In Murat, M. (éd.), L'allusion dans la littérature, Paris : Presses Universitaires de Paris Sorbonne, 209-235.

Authier-Revuz, J. \& Lala, M.-C. (2002). Avant-propos. In Authier-Revuz, J. \& Lala, M.-C. (éd.), Figures d'ajout. Phrase, texte, écriture, Paris : Presses Sorbonne Nouvelle, 7-12.

Badir, S. (2007). La sémiotique aux prises avec les médias. Semen, 23, 25-43.

Barthes, R. (1953, 1972). Le degré zéro de l'écriture. Paris : Seuil.

Batt, N. (1996). Du signe linguistique au signe littéraire : lire le complexe. In Costantini, M. \& Darrault-Harris, I. (éd.), Sémiotique, phénoménologie, discours, Paris : L'Harmattan, 125-133.

Bonhomme, M. (2003). Pour une approche pragmatico-cognitive des discours figuraux : l'exemple de l'allégorie. In Amossy, R. \& Maingeneau, D. (éd.), L'analyse du discours dans les études littéraires, Toulouse, Presses Universitaires du Mirail, 175-186.

Boucheron-Pétillon, S. (2002). Parenthèse et double tiret. In Authier-Revuz, J. \& Lala, M.-C. (éd.), Figures d'ajout. Phrase, texte, écriture, Paris : Presses Sorbonne Nouvelle, 123-130.

Butor, M. (1960/1964). Essais sur le roman. Paris : Gallimard.

Butor, M. (1962). Mobile. Paris : Gallimard.

Charaudeau, P. \& Maingeneau, D. (2002). Dictionnaire d'analyse du discours. Paris : Seuil.

Colas-Blaise, M. (2004). Le Discours rapporté du point de vue de la sémiotique : Dynamique discursive et avatars de la dénomination propre chez Patrick Modiano. In Lopez Munoz, J. M., Marnette, S. \& Rosier, L. (éd.), Le Discours rapporté dans tous ses états, Paris, L'Harmattan, 163-172.

Colas-Blaise, M. (2007). Éléments pour une poétique de l'entre-deux : une lecture sémiolinguistique d'Un balcon en forêt. In Reggiani, C. \& Stolz, C. (éd.), Du Bellay, Rotrou, Diderot, Verlaine, Gracq, Paris : Presses de l'Université Paris-Sorbonne, 127-141.

Drillon, J. (1991). Traité de la ponctuation française. Paris : Gallimard.

Fontanille, J. (1998). Sémiotique du discours. Limoges : PULIM.

Fontanille, J. (1999). Sémiotique et littérature. Essais de méthode. Paris : PUF.

Fontanille, J. (2003). Énonciation et modélisation. Modèles linguistiques, t. XXIV, fasc. 1, 109-133. 
Fontanille, J. (2006). Textes, objets, situations et formes de vie. Les niveaux de pertinence du plan de l'expression dans une sémiotique des cultures. In Alonso, J., Bertrand, D., Costantini, M. \& Dambrine, S. (éd.), La transversalité du sens. Parcours sémiotiques, Paris : Presses Universitaires de Vincennes, 213-240.

Fontanille, J. \& Zilberberg, C. (1998). Tension et signification. Sprimont : Pierre Mardaga.

Gardes-Tamine, J. (2004). Pour une grammaire de l'écrit. Paris : Belin.

Genette, G. (1991). Fiction et diction. Paris : Seuil.

Geninasca, J. (1997). La parole littéraire. Paris : PUF.

Gracq, J. (1989, 1995). Euvres complètes, t. 1 et t. 2. Paris : Gallimard (Bibliothèque de la Pléiade).

Grésillon, A. \& Maingeneau, D. (1984). Polyphonie, proverbe et détournement. Langages, 73, 112-124.

Guimier, C. (1996). Les adverbes du français : le cas des adverbes en -ment. Paris : Ophrys.

Helbo, A. (1975). Michel Butor. Vers une littérature du signe. Bruxelles : Éditions Complexe.

Herschberg Pierrot, A. (2005). Le style en mouvement. Paris : Belin.

Jaubert, A. (1990). La lecture pragmatique. Paris : Hachette.

Jaubert, A. (2003). Genres discursifs et genres littéraires : de la scène d'énonciation à l'empreinte stylistique. In Amossy, R. \& Maingeneau, D. (éd.), L'analyse du discours dans les études littéraires, Toulouse, Presses Universitaires du Mirail, 281-293.

Jaubert, A. (2005). Des styles au style : genres littéraires et création de valeur. In Gouvard, J.-M. (éd.), De la langue au style, Lyon : Presses Universitaires de Lyon, 37-50.

Jaubert, A. (2007). La diagonale du style. Etapes d'une appropriation de la langue. Pratiques, 135-136, 47-62.

Maingeneau, D. (1984). Genèses du discours. Liège : Pierre Mardaga.

Maingeneau, D. (1999). Ethos, scénographie, incorporation. In Amossy, R. (éd.), Images de soi dans le discours. La construction de l'ethos, Lausanne : Delachaux \& Niestlé, 75-100.

Maingeneau, D. (2003). Ouverture. Un tournant dans les études littéraires. In Amossy, R. \& Maingeneau, D. (éd.), L'analyse du discours dans les études littéraires, Toulouse, Presses Universitaires du Mirail, 15-25.

Maingeneau, D. (2004). Le discours littéraire. Paratopie et scène d'énonciation. Paris : Armand Colin.

Méchoulan, É. (2005) Présentation.

$<$ http://cri.histart.umontreal.ca/cri/fr/INTERMEDIALITES/presentation_texte.htm>

Metz, C. (1991). L'énonciation impersonnelle ou le site du film. Paris : Méridiens-Klincksieck.

Molinié, G. (1994). Le style en sémiostylistique. In Molinié, G. \& Cahné, P. (éd.), Qu'est-ce que le style ?, Paris : PUF, 201-211.

Molinié, G. (1998). Sémiostylistique. L'effet de l'art. Paris : PUF.

Rabatel, A. (2007). La dialectique du singulier et du social dans les processus de singularisation : style(s), idiolecte, ethos. Pratiques, 135-136, 15-34.

Riffaterre, M. (1971). Essais de stylistique structurale. Présentation et traductions de D. Delas. Paris : Flammarion.

Zilberberg, C. (2006). Éléments de grammaire tensive. Limoges : PULIM.

1 Pour une typologie des rapports entre le producteur et le récepteur, cf. M. Bonhomme (2003 : 180-181).

2 Au sujet de l'« objet textuel » et du texte, cf. Geninasca $(1997: 86):$ «Chaque usage, chaque "pratique discursive" a pour effet d'actualiser certaines des virtualités de cet objet textuel, par et à travers l'actualisation simultanée d'un 
sujet (une instance énonciative) et d'un objet (le texte proprement dit). Lire, interpréter un énoncé, en constituer la cohérence, cela revient à actualiser le texte - dont l'objet textuel n'est encore que la promesse - en vue de le saisir comme un tout de signification, comme un ensemble organisé de relations [...] ».

3 Voir aussi A. Rabatel (2007 : 24-25) : le «monisme permet de traiter de façon globale "le tout du style", selon une approche continuiste du phénomène linguistique de l'actualisation ». Il ajoute : " Ainsi le style est sans doute le lieu où se manifeste le mieux la dynamique de construction/spécification de soi à travers le retravail des formes sociales et culturelles par lesquelles les individus expriment leurs rapports entre eux, leur rapport au monde et leur rapport au langage ».

4 Cf. J. Fontanille (1999 : 194) : «Le style recouvre l'ensemble des faits textuels et discursifs grâce auxquels la praxis énonciative produit et reconnaît des effets d'identité ». Au sujet de l'identité, cf. aussi G. Achard-Bayle (2001).

5 Cf. A. Jaubert (2003: 288) au sujet de l'«émergence d'un effet de l'art que l'analyse stylistique tentera d'élucider».

6 Voir A. Jaubert (2007: 48) au sujet de la «complémentarité de la linguistique et de la stylistique en tant que démarches qui se prolongent l'une l'autre, et renvoient l'une à l'autre ».

7 Voir C. Metz (1991): "Les marques d'énonciation, même quand elles sont "naturelles", désignent l'activité discursive par un signal plus ou moins isolable, par un "geste", et ainsi s'en détachent [...]. [...] le style fait corps avec le discours et le teinte dans sa masse. [...] L'énonciation est une opération, le style une manière d'être » (cité par Fontanille, 1999 : 192).

8 Cf. M. Riffaterre (1971: 64) au sujet de la troisième propriété du contexte : le contexte « est variable et forme une série de contrastes avec les procédés stylistiques successifs. Seule cette variabilité peut expliquer pourquoi une unité linguistique acquiert, modifie ou perd son effet stylistique en fonction de sa position, pourquoi chaque écart à partir de la norme n'est pas nécessairement un fait de style et pourquoi effet de style n'implique pas anormalité ».

9 Cf. G. Molinié au sujet des régimes de littérarité «à intensité variable » et de la gradualité du style (1994: 202, 209).

10 On conçoit l'intérêt de la notion de «limite de rendement» introduite par Jaubert (2007: 54) : «La limite de rendement, c'est "l'heure de vérité" pour le signifié de puissance d'un fait de langue, le moment où, son potentiel étant révélé par une sollicitation extrême, il cristallise l'intelligibilité du discours, et par là se perçoit comme un fait de style, l'intelligibilité étant précisément la perception synthétique du sens et de la valeur ».

11 Cf. M. Bonhomme (2003 : 183-184) pour les trois sortes d'interprétations auxquelles donne lieu le texte allégorique.

12 Pour les "jugements d'identité » (individualité, tempérament, singularité, originalité), cf. J. Fontanille (1999: 196).

13 Les régimes de la réception peuvent être mis en relation avec les niveaux d'analyse qui permettent à A. Jaubert (2005: 40) de décrire l'objet de la stylistique comme un "objet à géométrie variable », en distinguant deux postulations, particularisante et universalisante, et « en échelonnant différentes saisies ».

14 Voir G. Genette (1991 : 151) : «Le style est bien dans les détails, mais dans tous les détails, et dans toutes leurs relations. Le "fait de style", c'est le discours lui-même » (cité par Adam, 1997 : 12).

15 Le style gagne à être articulé avec l'idiolecte qui, comme le souligne A. Rabatel (2007:27), «mieux que le style, permet de penser le singulier dans le langage $»$.

16 À ce sujet, voir aussi Colas-Blaise, 2004.

17 Voir J. Fontanille au sujet des trois facettes de la conversion connotative : «(i) la régularité d'une sélection, (ii) la corrélation avec d'autres isotopies, et (iii) la stabilisation intentionnelle du système semi-symbolique » (2003: 115). Comme le note J. Fontanille, la connotation n'est pas seulement une «dimension de l'analyse (ou une association d'idées de l'analyste), mais d'abord une propriété structurale et dynamique du texte lui-même » (ibid. : 114).

18 Selon J. Fontanille (2003 : 114), «l'analyse connotative "ferme" en quelque sorte le texte sur lui-même, en montrant que partout où il $\mathrm{y}$ a eu choix ou variation, émergent des figures qui constituent globalement un ensemble cohérent ». 
19 On considérera que le régime réflexif «méta-stylistique » renvoie aux «méta-sémiotiques ». Dans un dernier temps, J. Fontanille distingue celles-ci des «sémiotiques morpho-dynamiques », qui «comportent un principe de déformation interne et intrinsèque [...] : ce n'est pas l'invariant qu'elles schématisent, mais le principe de la variation dans son ensemble, et à cet égard, contrairement à ce qui se passe dans les méta-sémiotiques conceptuelles, l'invariant n'est qu'un cas particulier de l'ensemble de la structure dynamique » (2003: 122).

20 Ces points ont été développés dans Colas-Blaise, à paraître : «Comment opère le double tiret ? Éléments pour une grammaire du "décrochement" chez Julien Gracq ", Colloque international Les Linguistiques du détachement, Nancy, juin 2006. On y trouvera des arguments à l'appui d'une généralisation de la proposition comme forme canonique de la tirade.

21 Soit l'exemple suivant: «Je me sentais de connivence avec la pente de ce paysage glissant au dépouillement absolu. Il était fin et commencement. Au-delà de ces étendues de joncs lugubres s'étendaient les sables du désert, plus stériles encore ; et au-delà - pareils à la mort qu'on traverse - derrière une brume de mirage étincelaient les cimes auxquelles je ne pouvais plus refuser un nom. [...] un magnétisme secret m'orientait par rapport à la bonne direction" (Le Rivage des Syrtes, 1989: 611). La flexion de l'adjectif "pareils » disqualifie les tentatives d'appariement sur la base d'une construction appositive : elles entraîneraient la faillite de l'incidence canonique au sujet de la prédication principale. La restitution du syntagme nominal « les déserts » après la locution prépositionnelle « au-delà de » peut alors venir résoudre le conflit; la lecture peut également être orientée vers l'explicitation de la description définie «les sables» au poste de sujet et vers celle de la copule (« [les sables étaient] pareils à la mort qu'on traverse »). Dans les autres cas, le segment détaché est supposé assurer la continuité textuelle «à distance »: on considère que fort de son autonomie syntaxique, il défie la clôture graphique et enjambe les connecteurs spatial et temporel, pour ajouter à la caractérisation rhématique existante des informations au moins partiellement inédites.

22 Voir également Colas-Blaise, 2007.

23 À la base de la distinction entre les translations inter- et transmédiatiques, on peut situer la différence entre l'intersémiotique et la trans-sémiotique selon G. Molinié (1998 : 41, 44-45) : «L'inter-sémiotique, au sens restreint, ce qui veut dire au sens strict, désignerait l'étude des traces du traitement sémiotique d'un art dans la matérialité du traitement sémiotique d'un autre art »; quant à la démarche trans-sémiotique, soit il s'agit d'analyser « l'ensemble de la production d'un artiste [...] dans son unité stylistique à travers le traitement de matériaux sémiotiques différents », soit un «ensemble stylistique, un style, une manière esthétique prégnante » sont étudiés «à travers des réalisations sur matériaux sémiotiques différents ». 